\title{
Oxidation States of Graphene: Insights from Computational Spectroscopy
}

\author{
Wenhua Zhang ${ }^{1,2,3}$, Vincenzo Carravetta ${ }^{3}$, Zhenyu Li ${ }^{1,2} *$ Yi Luo $^{1,2} \oplus$ t† and Jinlong Yang ${ }^{1}$ \\ ${ }^{1}$ Hefei National Laboratory for Physical Sciences at Microscale, \\ University of Science and Technology of China, Hefei, Anhui 230026, China \\ ${ }^{2}$ Department of Theoretical Chemistry, School of Biotechnology, \\ Royal Institute of Technology, S-10691 Stockholm, Sweden and \\ ${ }^{3}$ Institute of Chemical Physical Processes, \\ CNR, via Moruzzi 1, 56124 Pisa, Italy
}

\begin{abstract}
When it is oxidized, graphite can be easily exfoliated forming graphene oxide (GO). GO is a critical intermediate for massive production of graphene, and it is also an important material with various application potentials. With many different oxidation species randomly distributed on the basal plane, GO has a complicated nonstoichiometric atomic structure that is still not well understood in spite of of intensive studies involving many experimental techniques. Controversies often exist in experimental data interpretation. We report here a first principles study on binding energy of carbon $1 s$ orbital in GO. The calculated results can be well used to interpret experimental X-ray photoelectron spectroscopy (XPS) data and provide a unified spectral assignment. Based on the first principles understanding of XPS, a GO structure model containing new oxidation species epoxy pair and epoxy-hydroxy pair is proposed. Our results demonstrate that first principles computational spectroscopy provides a powerful means to investigate GO structure.
\end{abstract}

*E-mail: zyli@ustc.edu.cn

†E-mail: luo@kth.se 


\section{INTRODUCTION}

Graphene is one of the most studied materials in recent years owing to its peculiar physical properties and great potential for various applications. [1, 2, 3] A big technological challenge in graphene research is to massively produce high quality samples. Epitaxial growth represents a suitable way for electronics application. [4, 5, 6, 7] However, for general purpose applications, solution based strategy is more attractive. One promising graphene synthesis route is oxidizing and exfoliating graphite, then reducing the obtained graphene oxide (GO) sheets. [8, 9, 10, 11, 12] By this way, the pristine graphene properties can be largely restored during the reduction. At the same time, GO itself is an important material with various applications. It is a good candidate for composite materials, [13] and its hydrate, GO paper, shows excellent mechanic properties. [14] It is possible to integrate GO into all-graphene electronic devices [15] and to obtain near-UV to blue photoluminescence from GO. [16] GO based hydrogen storage materials are suggested to be very promising. [17, 18, 19] By using GO support, Scheuermann et al. [20] also achieved much higher Pd catalysis activity compared to conventional $\mathrm{Pd} / \mathrm{C}$ system.

Because of its importance, it is highly desirable to understand the atomic structure of GO and the oxidation processes to prepare it. There is a long history of GO structure studies. [21, 22, 23, 24, 25, 26] In the most popular structure model, GO is constituted of epoxy and hydroxy groups attached to the carbon layer, and terminated with hydroxy and carboxyl groups. Many experimental techniques, such as nuclear magnetic resonance (NMR), [25, 26, 27, 28] x-ray photoemission spectroscopy (XPS), [26, 29, 30, 31, 32] and vibrational spectroscopy, [33, 34] have been employed to explore the structure information. However, the interpretation of experimental data turns out to be difficult, since GO is a nonstoichiometric compound with randomly distributed oxidation groups.

XPS is one of the best local structure probes to identify oxidation species on GO. Experimental XPS for heavily oxidized GO have mainly three peaks, namely $P_{1}, P_{2}$, and $P_{3}$ from lower to higher binding energies. It is commonly accepted that $P_{1}$ comes from $s p^{2}$ carbon, and $P_{2}$ is contributed by carbon atoms connected to epoxy (C-E) and hydroxy $(\mathrm{C}-\mathrm{OH})$ groups. However, definitive assignment for $P_{3}$ is currently not available. Szabo et al. [26] assigned this peak to ketones $(\mathrm{C}=\mathrm{O})$, while Jeong et al. [29] attributed it to edge carboxyl $(\mathrm{COOH})$ groups. Considering its high intensity, peak $P_{3}$ can not be solely contributed by 
edge groups. On the other hand, the existence of interior ketone species has been excluded by other experiments. For example, a recent two dimensional NMR experiment suggested that carbonyl groups are spatially separated from the majority $s p^{2}, \mathrm{C}-\mathrm{OH}$, and C-E carbons. [27] This result implies that $\mathrm{C}=\mathrm{O}$ groups mainly distribute at $\mathrm{GO}$ edges, as also suggested by a recent $\mathrm{O}$ 1s X-ray absorption spectrum measurement. [35] Therefore, both experimental XPS assignments are questionable, and new species may exist in GO [36] that has a contribution to $P_{3}$.

Recently, new functional groups such as epoxy pair (EP) and manganate ester $\left(\mathrm{C}-\mathrm{MnO}_{4}^{-}\right)$ have been proposed in studies on oxidation induced cut of graphitic materials. [36, 37] When epoxy chain breaks underlying C-C bonds, [38] additional epoxy groups can attach to it forming EP groups. EP can then transit to carbonyl pair (CP) and finally break the carbon network. However, there is a significant dissociation barrier for the first EP to CP transition, [36] which makes EP can in principle be widely existed in GO. Besides these two groups, to reach a tentative assignment of NMR spectrum, a new structure motif of lactol rings is suggested to be existed at GO edge sites. [28] However, XPS signatures of these new spices are still unclear.

Theoretical simulation can provide useful information to obtain correct spectroscopic assignments and thus a more reliable GO structure model. Unfortunately, previous theoretical studies on GO mainly focus on energetics [36, 39, 40, 41, 42] instead of properties that can be directly compared with experimental data. The complexity of the GO potential energy surface limits the power of energetics study to some extent, especially when artificial periodic boundary condition must be adopted. Kudin et al. [33] have simulated the Raman spectra of GO, and proposed an alternating single-double carbon bond model. Compared to Raman spectroscopy, XPS is a more localized probe and thus a better choice to study local structures.

In this study, we calculate core chemical shift of carbon $1 s$ orbital for possible oxidation species in GO using density functional theory, which can be compared to experimental XPS spectra directly and leads to a unified reassignment. Based on such a computational spectroscopy strategy, we propose a GO structure model with new species epoxy pair and hydroxy-epoxy pair. The remainder of this article is organized as follows. In section 2, we introduce the computational methods. XPS simulation results and implications to GO structure are present in section 3. Finally, we conclude in section 4. 


\section{COMPUTATIONAL DETAILS}

XPS simulation with plane wave basis set. Spin polarized electronic structure calculations are performed with the Vienna ab-initio simulation package (VASP) [43] using Perdew, Burke and Ernzerhof (PBE) [44] exchange-correlation functional. The electron-ion interaction is described with projector augmented wave (PAW) method [45], which permits to set the occupation of core level as any number between 0 and 1 . Different two dimensional sheet and one dimensional nanoribbon models are adopted to simulate GO. The binding energy of $\mathrm{C} 1 s$ orbital is calculated as the energy difference between the ground state and the core-excited state with one core electron removed. [46] We have tested this computational approach by calculating the $1 s$ core orbital binding energies of $\mathrm{C}, \mathrm{N}$, and $\mathrm{O}$ atoms in alanine and tryptophan, and the obtained core chemical shifts in different chemical environments agree well with the corresponding experimental values. [47]

XPS simulation with atomic basis set. Cluster models are also used to calculated the binding energy of $\mathrm{C} 1 s$ orbital. The geometries of all clusters are optimized with $\mathrm{DMol}^{3}$ [48, 49] and the binding energy calculations are performed with StoBe-deMon. [50] In binding energy calculations, gradient corrected Becke (B88)[51] exchange functional and Perdew (P86) [52] correlation functional are adopted. Effective core potential is used to describe the non excited atoms and for ionized atoms, IGLO-III basis set [53] is used instead. To get the binding energy of core electron, one $\mathrm{C} 1 s$ electron is removed from the system. The core chemical shift is defined as the energy difference between core electron removed system and the ground state. Such a XPS simulation protocol has been widely used in many studies, and it generally leads to a good agreement with experiment. [54, 55, 56] For different cluster models, the core chemical shift is calibrated according to the binding energy of C-H far way from the oxidation groups.

\section{RESULTS AND DISCUSSION}

The main task of this study is to calculate the core chemical shift of $\mathrm{C} 1 \mathrm{~s}$ orbital for different oxidation species. For this purpose, two first principles simulation protocols and many different structure models are used. The absolute value of core chemical shift can be affected by many factors, for examples, by charging effect in experiment and by work function 
of the theoretical GO model. We find that for a perfect graphene sheet, the work function has a value of $4.7 \mathrm{eV}$, while for a hydrogen saturated 1D graphene nanoribbon, it becomes $3.7 \mathrm{eV}$. It indicates that the structure model used for GO can have a significant effect on the work function and thus on the absolute value of core chemical shift. Therefore, we only focus on relative core chemical shift (R-CCS). The average binding energy of epoxide group in each structure model is set as a common reference to compare results from calculations and from experiments.

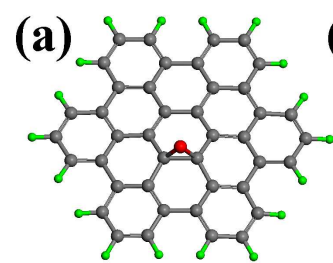

(b)
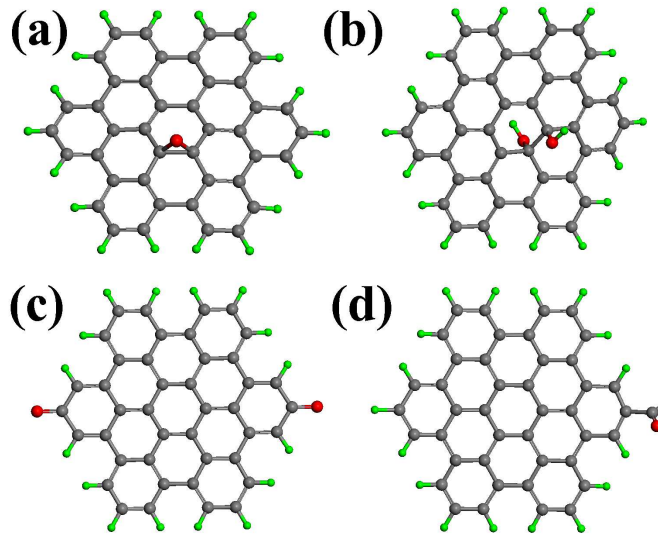

(d)
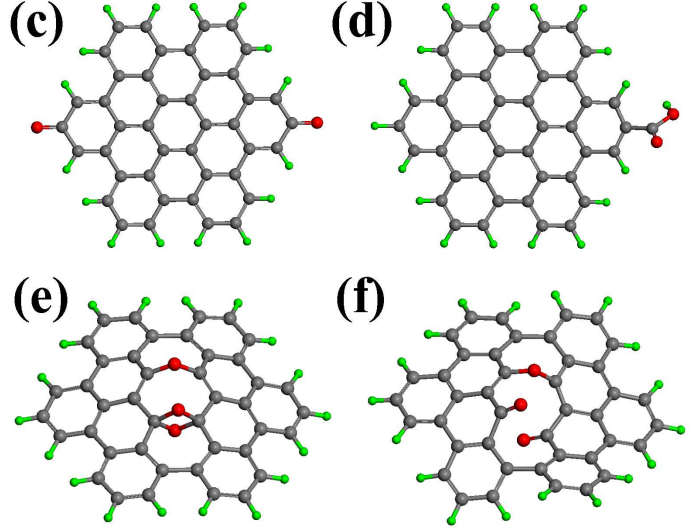

(f)
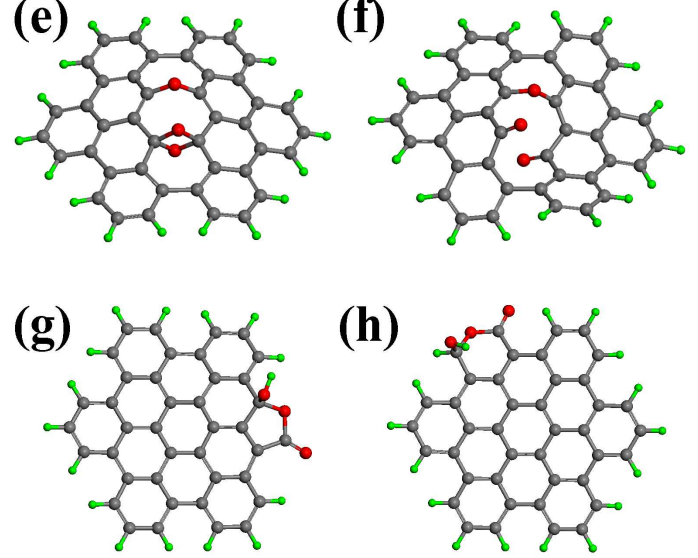

(h)

(i)
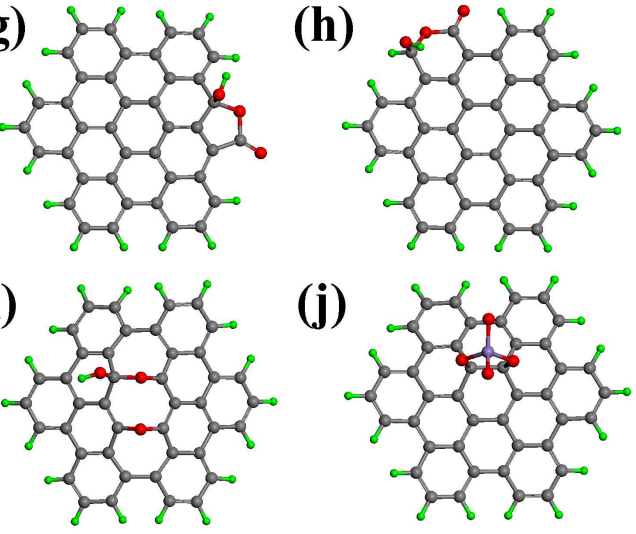

(j)

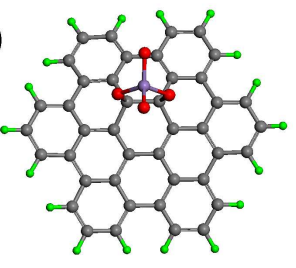

FIG. 1: Cluster models used to calculate C 1s binding energies. (a) epoxy, (b) two OH groups, (c) two edge $\mathrm{C}=\mathrm{O}$ groups, (d) carboxyl group, (e) line defect epoxy group $\left(\mathrm{C}-\mathrm{E}^{\prime}\right)$ and epoxy pair (C-EP) group, (f) C-E' and carbonyl pair (CP), (g) five-ring lactol group, (h) six-ring lactol group, (i) hydroxy-epoxy pair, and (j) MnO4- attached structure. 
We first consider the well studied epoxy and hydroxyl groups, which contribute to XPS peak $P_{2}$. In some experiments, $P_{2}$ is further deconvoluted into two sub-peaks, namely $P_{2}^{a}$ and $P_{2}^{b}$. [29, 31] The former is about $0.8 \mathrm{eV}$ lower in binding energy than the latter. These two peaks have been tentatively assigned to $\mathrm{C}-\mathrm{OH}$ and $\mathrm{C}-\mathrm{E}$, respectively. However, such an assumption needs more stringent test, for example, by first-principles calculation on the binding energy of carbon $1 s$ orbital.

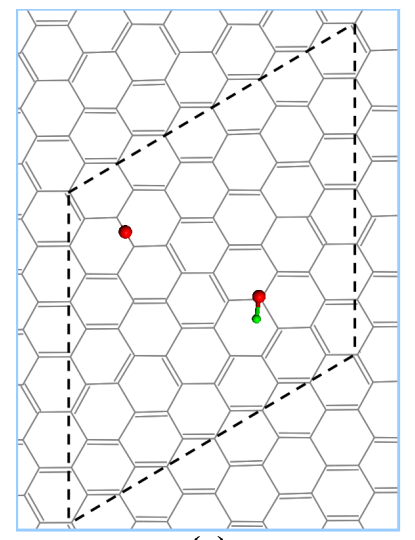

(a)

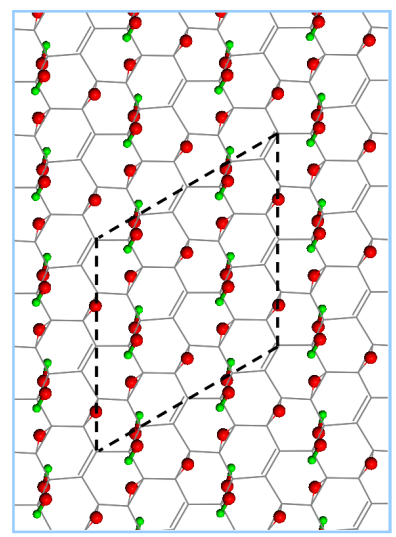

(b)

FIG. 2: (a) Graphene sheet with isolated epoxy and hydroxy groups. Dashed lines mark a $(6 \times 6)$ unit cell. (b) A GO model with epoxy and hydroxy groups. Dashed lines indicate the $(4 \times 4)$ cell used in our calculations. Red and green spheres represent oxygen and hydrogen atoms, respectively.

As shown in Figure 1a and 1 $\mathrm{b}$, we construct two clusters, one with C-E and the other with $\mathrm{C}-\mathrm{OH}$. Both clusters have an exclusive Clar's structure, where all $\pi$-electrons form separated aromatic sextets. [57] Dangling bonds are saturated with hydrogen, and the oxidation groups are put in the center of the cluster. The calculated R-CCS of C-OH with respect to C-E is only $0.1 \mathrm{eV}$, indicating that $\mathrm{C}-\mathrm{E}$ and $\mathrm{C}-\mathrm{OH}$ are difficult to be resolved in XPS. It is also interesting to note that the core binding energy of $\mathrm{C}-\mathrm{OH}$ is larger than that of C-E, contrary to the tentative assignment for $P_{2}^{a}$ and $P_{2}^{b}$.

For periodic systems, we put two isolated hydroxy and epoxy groups in a big $(6 \times 6)$ supercell, as shown in Figure 2a. R-CCS of $s p^{2} \mathrm{C}$ with respect to $\mathrm{C}-\mathrm{E}$ is $-1.7 \mathrm{eV}$, while the difference between the binding energies of $\mathrm{C}-\mathrm{E}$ and $\mathrm{C}-\mathrm{OH}$ is still within $0.1 \mathrm{eV}$, consistent with the atomic basis set calculation. To take the coupling between epoxy and hydroxy groups into account, we also consider a more realistic GO structure model [40] (Figure 2b). In this case, the binding energy of $\mathrm{C}-\mathrm{OH}$ is larger than that of $\mathrm{C}-\mathrm{E}$ by $0.3 \mathrm{eV}$, showing 
the important effect of chemical environment. However, such a small difference is still not distinguishable in XPS experiments. [26, 30] Therefore, a new assignment for $P_{2}^{a}$ and $P_{2}^{b}$ is required.

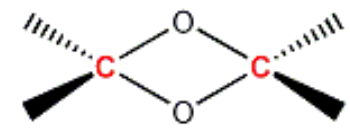

(a)

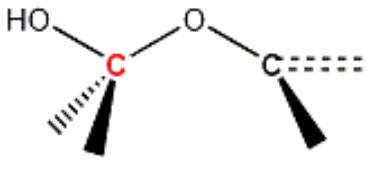

(b)

FIG. 3: New oxidation species. (a) epxoy pair, (b) hydroxy-epxoy pair. The corresponding carbon atoms (red) are named as C-EP and E-C-OH, respectively. Both C-EP and E-C-OH are monovalently connected to two oxygen atoms.

To better understand the XPS peaks, more oxidation species beyond C-OH and C-E should be considered. First, we take edge groups. It is known that there are $\mathrm{C}=\mathrm{O}, \mathrm{COOH}$, and hydroxy (C-OH-edge) groups existing at GO edge. In this study, we also consider other edge groups including $\mathrm{C}-\mathrm{H}, \mathrm{CH}_{3}, \mathrm{CO}_{3}$, and lactol (Figure $1 \mathrm{~g}$ and $1 \mathrm{~h}$ ). For new interior groups, some clues can be found from the oxidation induced cutting of graphene. [36] In highly oxidized GO, some epoxy groups may align in a line, which leads to the break of the underlying C-C bonds. [38] Then, a new epoxy group can be attached to form a EP group (C-EP, Figure 4a), [36] or a hydroxy can be added to from a epoxy-hydroxy pair (E-C-OH, Figure 4b). E-C-OH is actually also existed in the recently proposed edge lactol groups, [28] and the latter can be considered as a special case of the former.

Once the wide existence of aligned epoxy groups is recognized, the formation of C-EP and E-C-OH becomes very natural. When aligned epoxy groups break the underlying C-C bonds, the corresponding carbon atoms ( $\alpha$ carbon) are connected to two other carbon atoms ( $\beta$ carbon) and the epoxy group, to which the $\alpha$ carbon is bound with one valence electron. Therefore, there is a $\pi$ electron left on the $\alpha$ carbon. If both the two $\beta$ carbon atoms are oxidized to be $s p^{3}$ hybridized, then the $\alpha$ carbon becomes a radical. In highly oxidized samples, the possibility that $\beta$ carbon atoms are saturated is quite high. Therefore, the $\alpha$ carbon is very possible to be attacked to form epoxy pair or epxoy-hydroxy pair groups in the oxidizing environment.

Together with C-E and C-OH, these new oxidation species have been put in various of structure models. Individual groups have been put on clusters (Figure11). We also construct 

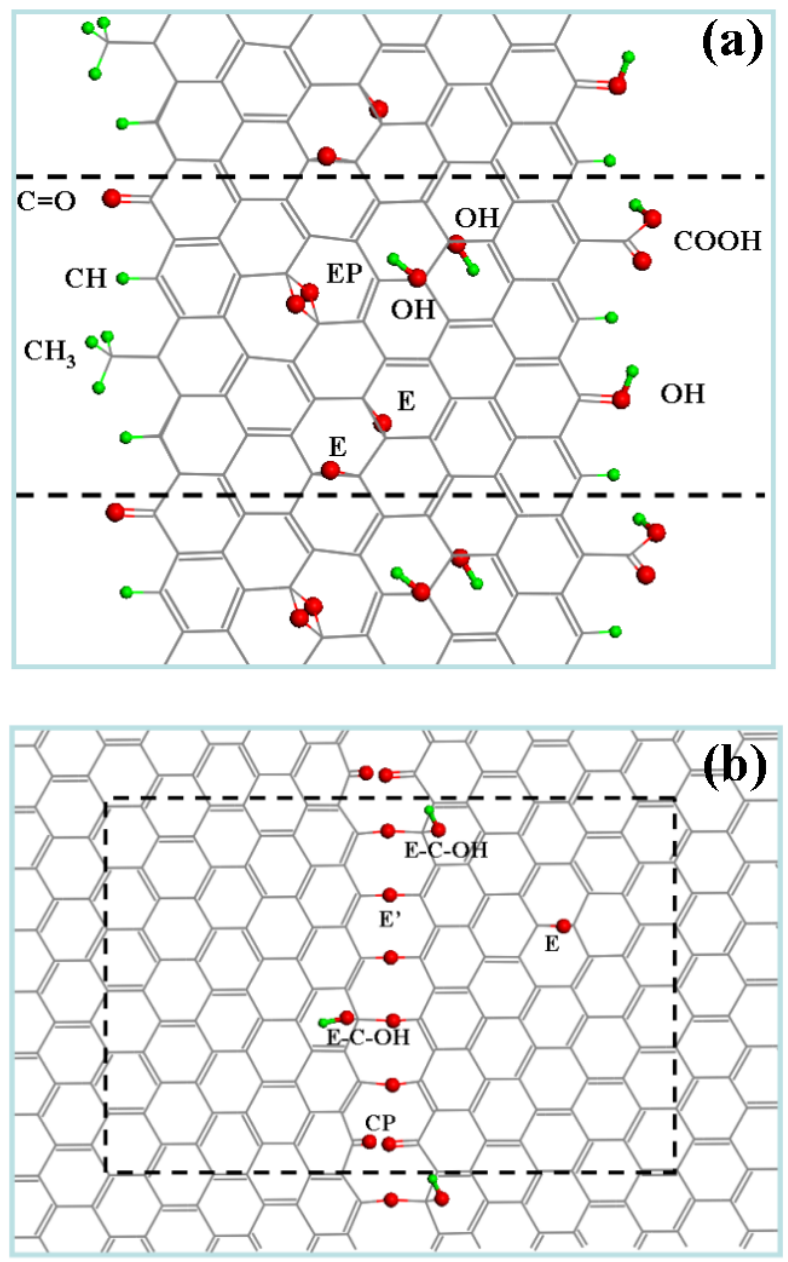

FIG. 4: (a) A GO nanoribbon model with zigzag edges. The part between two dashed lines is a unit cell along the ribbon direction. (b) A GO structure model based on epoxy line defect.

several GO nanoribbon models with many oxidation species randomly distributed both on the plane and at the edges. One of these ribbon models with zigzag edges is shown in Figure 4a. It turns out that the calculated core chemical shifts are almost not dependent on whether zigzag or armchair edges are adopted in the GO model. Another kind of GO models we considered is epoxy line defect based, for which different functional groups are put on the epoxy chain, as shown in Figure $4 \mathrm{~b}$.

With these GO models, we have calculated the core chemical shift for oxidation species C$\mathrm{OH}, \mathrm{C}-\mathrm{E}, \mathrm{C}-\mathrm{EP}$, and E-C-OH on planes, and $\mathrm{C}-\mathrm{OH}-\mathrm{edge}, \mathrm{C}=\mathrm{O}, \mathrm{C}-\mathrm{H}, \mathrm{CH}_{3}, \mathrm{COOH}, \mathrm{COOO}$, and lactol at edges. The calculated R-CCS values are collected in Table I together with experimental values reported in the literature. [26, 29] Chemical shifts for all calculated 
oxidation species are well within the energy window of the experimental XPS data. Again, we find that epoxy and hydroxy groups in the interior of ribbons are not distinguishable. The peak $P_{2}^{a}$ originally assigned to $\mathrm{C}-\mathrm{OH}$ by Jeong et al. [29] has contributions from edge attached $\mathrm{C}-\mathrm{OH}$-edge and $\mathrm{C}=\mathrm{O}$.

We notice that, in experimental spectra, $P_{2}^{a}$ have comparable intensity to $P_{2}^{b}$. Therefore, there should be other contributions to $P_{2}^{a}$ besides the edge groups. For epoxy chains with the underlying $\mathrm{C}-\mathrm{C}$ bonds broken, the corresponding carbon atoms (C-E') almost keep $s p^{2}$ hybridization with a C-O bond length about $1.37 \AA$, very close to that in C-OH-edge (1.36 $\AA)$. R-CCS of $\mathrm{C}^{-\mathrm{E}^{\prime}}$ is also very similar to that of $\mathrm{C}-\mathrm{OH}$-edge. Since it is distributed in the interior part of $\mathrm{GO}, \mathrm{C}-\mathrm{E}^{\prime}$ can make a strong peak in XPS.

Our calculations show both C-EP and E-C-OH contribute to the XPS peak $P_{3}$. R-CCS of C-EP in an epoxy line defect $(1.5 \mathrm{eV})$ is slightly higher than its isolated counterparts $(0.9$ to $1.0 \mathrm{eV}$ ). E-C-OH has a larger core level binding energy compared to C-EP. Edge group $\mathrm{COOH}$ has a similar R-CCS with C-EP. Since the newly proposed five- or six-membered lactol ring contain E-C-OH, its R-CCS also locates in the region of $P_{3}$, only slightly lower than that of interior hydroxy-epoxy pair. However, as an edge groups like $\mathrm{COOH}$, it only has limited contribution to XPS. Main contribution to $P_{3}$ thus comes from C-EP and E-C-OH.

EP can dissociate to form a carbonyl pair (CP, Figure 1f and 4b). [36] R-CCS of an individual $\mathrm{CP}$ is close to zero, significantly higher than edge $\mathrm{C}=\mathrm{O}$ group, indicating its chemical environment is strongly affected by neighboring $\mathrm{C}-\mathrm{E}^{\prime}$. If the whole line defect is broken, $\mathrm{CP}$ becomes $\mathrm{C}=\mathrm{O}$ edge groups with a negative $\mathrm{R}-\mathrm{CCS}$. Since an existing $\mathrm{CP}$ strongly lowers the dissociation barrier of neighboring EPs, isolated CPs are not expected to be widely existed.[36] However, an important fact disclosed here it that, even if $\mathrm{C}=\mathrm{O}$ groups exist in the interior of GO, its contribution to XPS goes to $P_{2}$ instead of the originally supposed $P_{3}$. [26]

Another spices mentioned in the literature is $\mathrm{C}^{-\mathrm{MnO}_{4}^{-}}$. In our VASP calculations with plane wave basis set, for both a $(6 \times 6)$ unitcell and a cluster model, we fail to get a local minimum structure with $\mathrm{MnO}_{4}^{-}$adsorbed on graphene, $\mathrm{MnO}_{4}^{-}$always moves away during the optimization. This result suggests that $\mathrm{C}-\mathrm{MnO}_{4}^{-}$may be not stable or only stable in solution, and thus does not exist during XPS measurements. An adsorbed structure can be obtained using the atomic basis set with the $\mathrm{DMol}^{3}$ package. With this geometry, the calculated R-CCS is about $-0.7 \mathrm{eV}$, far away from peak $P_{3}$. 
TABLE I: R-CCS of carbon $1 s$ orbital for various oxidation species reported in experiment and calculated with atomic and plane wave basis sets in this work. The binding energy of C-E $(287.2 \mathrm{eV})$ is set as a common reference point. The numbers in parentheses are the linewidths of experimental peaks.

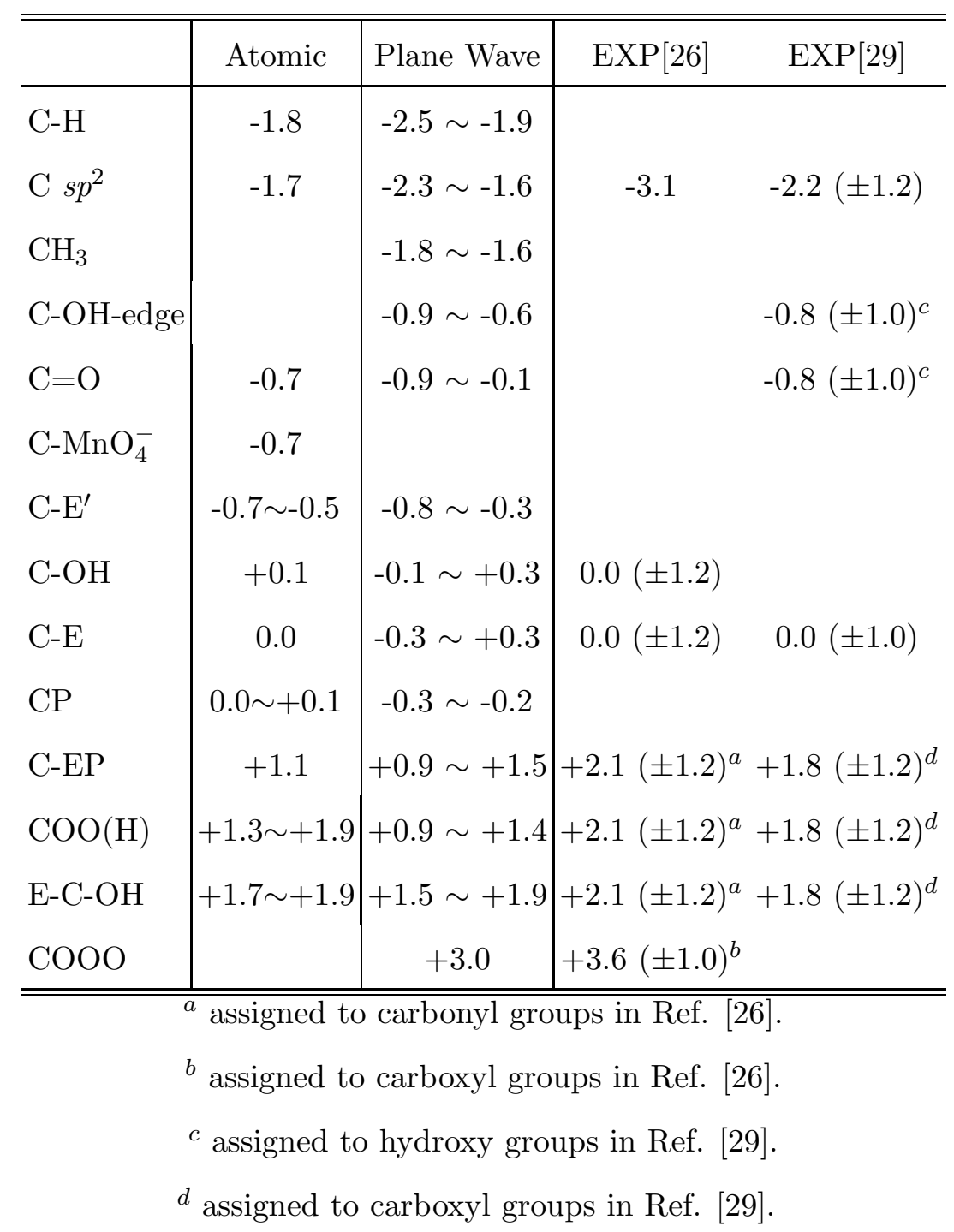

In some experimental XPS data, there is a small peak with a R-CCS more than $3 \mathrm{eV}$.[26] Our calculations suggest that it can be related to carbon atom connected to three oxygen, possibly at GO edges. [47] Until now, as shown in Figure 5, we reach a unified assignment for experimental XPS. The lowest peak $P_{1}$ comes from $s p^{2}$ carbon. Peak $P_{2}$ has two contributions: the lower one comes from edge groups and edge-like group $\mathrm{C}-\mathrm{E}^{\prime}$, and the higher one is contributed by epoxy and hydroxy groups. Peak $P_{3}$ mainly comes from C-EP and E-C-OH, with also small contributions from edge $\mathrm{COOH}$ groups. 


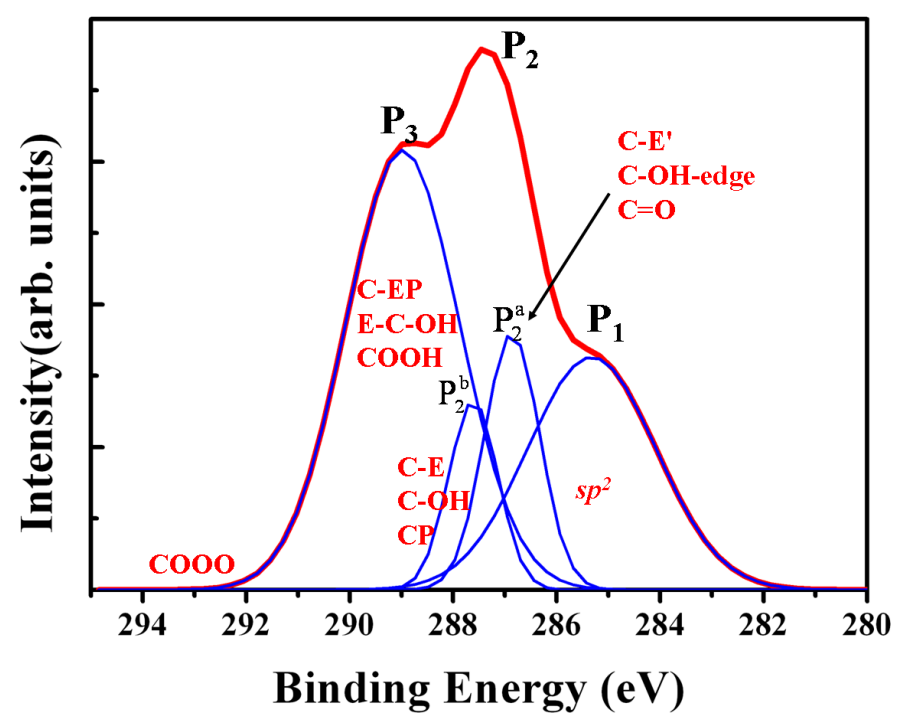

FIG. 5: Schematic experimental XPS curve and our first principles assignment.

With the proper first-principles assignments, the XPS intensity evolution observed in experiments [26, 29] can also be well understood. First of all, when the degree of oxidation increases, the peak of $s p^{2}$ carbon $\left(P_{1}\right)$ should decrease or even disappear for completely oxidized samples. At the same time, an increase of $P_{3}$ compared to $P_{2}$ was observed experimentally, [26, 29] which is because more EP and E-C-OH groups appear during the oxidation. Jeong et al. [29] observed an increase of $P_{2}^{a}$ and a decrease of $\mathrm{P}_{2}^{b}$ during oxidation, which can also be understood with our assignments. In fact, during the increase of the degree of oxidation, the possibility for epoxy groups to align in a line increases, which leads to more C-E' groups $\left(P_{2}^{a}\right)$. When GO is heated, a significant decrease of $P_{3}$ has been observed. [29] The dissociation of EP to CP should have made an important contribution to it.

\section{CONCLUSIONS}

Our DFT calculations provide relative C $1 s$ core chemical shifts for possible oxidation species on GO, whose binding energy follows the order: $\mathrm{C} s p^{2}<$ edge groups ( $\mathrm{such}$ as $\mathrm{C}=\mathrm{O}$, $\mathrm{C}-\mathrm{OH}-\mathrm{edge}$, and $\left.\mathrm{C}-\mathrm{E}^{\prime}\right)<\mathrm{C}-\mathrm{OH}$ and $\mathrm{C}-\mathrm{E}<\mathrm{C}-\mathrm{EP}, \mathrm{COOH}$, and $\mathrm{E}-\mathrm{C}-\mathrm{OH}<\mathrm{COOO}$. These results lead to a reassignment of experimental XPS spectra: (1) $P_{2}$ is mainly contributed by $\mathrm{C}-\mathrm{E}$ and $\mathrm{C}-\mathrm{OH}$, and they are not distinguishable; (2) edge groups and edge-like $\mathrm{C}-\mathrm{E}^{\prime}$ 
groups has lower binding energies compared to other $P_{2}$ groups; (3) although it has been previously used to explain $P_{3}$ in XPS experiments, $\mathrm{C}=\mathrm{O}$ actually has a low binding energy corresponding to $P_{2}$; (4) $P_{3}$ mainly comes from new species C-EP and E-C-OH; (5) even higher binding energy may obtained from COOO.

Based on our first principles spectroscopic understanding, we reach the following two important conclusions about the atomic structure of GO: (1) epoxy and hydroxy groups can be closely packed together forming epoxy pairs and epoxy-hydroxy pairs in highly oxidized samples; (2) as the main species contributed to the lower energy part of $P_{2}$, aligned epoxy group $\left(\mathrm{C}-\mathrm{E}^{\prime}\right)$ represents an important GO structure motif, which should be considered in all GO property studies and not only in oxidation induced cutting. Theoretical simulations also lead to an understanding of the experimental evolution of XPS for different samples, which provides us some insights to the oxidation processes of GO. Our results demonstrate that first principles computational spectroscopy is a very promising tool in nano structure research.

\section{Acknowledgments}

This work is partially supported by NFSC (20803071, 20933006, 50721091), by MOE (FANEDD-2007B23, NCET-08-0521), by CAS (KJCX2-YW-W22), by the National Key Basic Research Program (2006CB922004), and by USTC-SCC, SCCAS, and Shanghai Supercomputer Center.

[1] A. K. Geim and K. S. Novoselov, Nat. Mater. 6, 183 (2007).

[2] A. H. Castro Neto, F. Guinea, N. M. R. Peres, K. S. Novoselov and A. K. Geim, Rev. Mod. Phys. 81, 81, 109 (2009).

[3] A. K. Geim, Science 324, 1530 (2009).

[4] C. Berger, Z. Song, X. Li, X. Wu, N. Brown, C. Naud, D. Mayou, T. Li, J. Hass, A. N. Marchenkov, E. H. Conrad, P. N. First and W. A. de Heer, Science 312, 1191 (2006).

[5] P. W. Sutter, J.-I. Flege and E. A. Sutter, Nature Mater. 7, 406 (2008).

[6] K. S. Kim, Y. Zhao, H. Jang, S. Y. Lee, J. M. Kim, K. S. Kim, J.-H. Ahn, P. Kim, J.-Y. Choi and B. H. Hong, Nature 457, 706 (2009). 
[7] X. Li, W. Cai, J. An, S. Kim, J. Nah, D. Yang, R. Piner, A. Velamakanni, I. Jung, E. Tutuc, S. K. Banerjee, L. Colombo and R. S. Ruoff, Science 324, 1312 (2009).

[8] S. Stankovich, D. A. Dikin, C. H. B. Dommett, K. M. Kohlhaas, E. J. Zimney, E. A. Stach, R. D. Piner, S. T. Nguyen and R. S. Ruoff, Nature 442, 282 (2006).

[9] M. J. McAllister, J.-L. Li, D. H. Adamson, H. C. Schniepp, A. A. Abdala, J. Liu, M. HerreraAlonso, D. L. Milius, R. Car, R. K. Prud'homme and I. A. Aksay, Chem. Mater. 19, 4396 (2007).

[10] V. C. Tung, M. J. Allen, Y. Yang and R. B. Kaner, Nat. Nanotech. 4, 25 (2008).

[11] G. Eda, G. Fanchini and M. Chhowalla, Nat. Nanotech. 3, 270 (2008).

[12] S. Park and R. S. Ruoff, Nat. Nanotech. 4, 217 (2009).

[13] T. Ramanathan, A. A. Abdala, S. Stankovich, D. Dikin, M. Herrera-Alonso, R. D. Piner, D. H. Adamson, H. C. Schniepp, X. Chen, R. S. Ruoff, S. T. Nguyen, I. A. Aksay, R. K. PrudHomme and L. C. Brinson, Nat. Nanotech. 3, 327 (2008).

[14] D. A. Dikin, S. Stankovich, E. J. Zimney, R. D. Piner, G. H. B. Dommett, G. Evmenenko, S. T. Nguyen and R. S. Ruoff, Nature 448, 457 (2007).

[15] X. Wu, M. Sprinkle, X. Li, F. Ming, C. Berger and W. A. de Heer, Phys. Rev. Lett. 101, 026801 (2008).

[16] G. Eda, Y.-Y. Lin, C. Mattevi, H. Yamaguchi, H.-A. Chen, I.-S. Chen, C.-W. Chen and M. Chhowalla, Adv. Mater. in press. (preprint available at http://arxiv.org/abs/0909.2456 (2009).)

[17] L. Wang, F. H. Yang, R. T. Yang and M. A. Miller, Ind. Eng. Chem. Res. 48, 2920 (2009).

[18] L. Wang, K. Lee, Y.-Y. Sun, M. Lucking, Z. Chen, J. J. Zhao and S. B. Zhang, ACS Nano ASAP article. DOI:10.1021/nn900667s (2009),

[19] G. M. Psofogiannakis and G. E. Froudakis, J. Am. Chem. Soc. ASAP article. DOI:10.1021/ja906159p (2009).

[20] G. M. Scheuermann, L. Rumi, P. Steurer, W. Bannwarth and R. Mulhaupt, J. Am. Chem. Soc. 131, 8262 (2009).

[21] U. Hofmann and R. Holst, Ber. Dtsch. Chem. Ges. 72, 754 (1939).

[22] G. Ruess, Monatsch. Chem. 76, 381 (1946).

[23] W. Scholz and H.-P. Z. Boehm, Anorg. Allg. Chem. 369, 327 (1969).

[24] T. Nakajima and Y. Matsuo, Carbon 32, 469 (1994). 
[25] A. Lerf, H. He, M. Forster and J. Klinowski, J. Phys. Chem. B 102, 4477 (1998).

[26] T. Szabó, O. Berkesi, P. Forgó, K. Josepovits, Y. Sanakis, D. Petridis and I. Dékány, Chem. Mater. 18, 2740 (2006).

[27] W. Cai, R. D. Piner, F. J. Stadermann, S. Park, M. A. Shaibat, Y. Ishii, D. Yang, A. Velamakanni, S. J. An, M. Stoller, J. An, D. Chen and R. S. Ruoff, Science 321, 1815 (2008).

[28] W. Gao, L. B. Alemany, L. Ci and P. M. Ajayan, Nat. Chem. 1, 401 (2009).

[29] H.-K. Jeong, Y. P. Lee, R. J. W. Lahaye, M.-H. Park, K. H. An, I. J. Kim, C.-W. Yang, C. Y. Park, R. S. Ruoff and Y. H. Lee, J. Am. Chem. Soc. 130, 1363 (2008).

[30] X. B. Fan, W. C. Peng, Y. Li, X. Y. Li, S. L. Wang, G. L. Zhang and F. B. Zhang, Adv. Mater. 20, 4490 (2008).

[31] S. J. Park, K. S. Lee, G. Bozoklu, W. W. Cai, S. T. Nguyen and R. S. Ruoff, ACS Nano. 2, $572(2008)$.

[32] D. Yang, A. Velamakanni, G. Bozoklu, S. Park, M. Stoller, R. D. Piner, S. Stankovich, I. Jung, D. A. Field, C. A. Ventric Jr. and R. S. Ruoff, Carbon 47, 145 (2009).

[33] K. N. Kudin, B. Ozbas, H. C. Schniepp, R. K. Prud'homme, I. Aksay and R. Car, Nano Lett. 8, 36(2008).

[34] C. Hontoria-Lucas, A. J. Lopez-Peinado, J. D. Lopez-Gonzalez,M. L. Rojas-Cervantes and R. M. Martin-Aranda, Carbon 133, 1585 (1995).

[35] H.-K. Jeong, H.-J. Noh, J.-Y. Kim, M. H. Jin, C. Y. Park and Y. Lee, Euro. Phys. Lett. 82, 67004 (2008).

[36] Z. Li, W. Zhang, Y. Luo, J. Yang and J. G. Hou, J. Am. Chem. Soc. 131, 6320 (2009).

[37] D. V. Kosynkin, A. L. Higginbotham, A. Sinitskii, J. R. Lomeda, A. Dimiev, B. K. Price and J. M. Tour, Nature 458, 872 (2009).

[38] J.-L. Li, K. N. Kudin, M. J. McAllister, R. K. PrudHomme, I. A. Aksay and R. Car, Phys. ReV. Lett. 96, 176101 (2006).

[39] J. T. Paci, T. Belytschko and G. C. Schatz, J. Phys. Chem. C 111, 18099 (2007).

[40] D. W. Boukhvalov and M. I. Katsnelson, J. Am. Chem. Soc. 130, 10697 (2008).

[41] X, Gao, L. Wang, Y. Ohtsuka, D.-E. Jiang, Y. Zhao, S. Nagase and Z. Chen, J. Am. Chem. Soc. 131, 9663 (2009).

[42] J. A. Yan, L. Xian and M. Y. Chou, Phys. Rev. Lett. 103, 086802 (2009).

[43] G. Keresse and J. Hafner, Phys. Rev. B 47, 558 (1993). 
[44] J. Perdew, K. Burke and M. Ernzerhof, Phys. Rev. Lett. 77, 3865 (1996).

[45] G. Kresse and D. Joubert, Phys. Rev. B 59, 1758 (1999).

[46] L. Köhler and G. Kresse, Phys. Rev. B 70, 165405 (2004).

[47] Experimental XPS data, test calculations, other constructed GO models, and all calculated R-CCS data. See EPAPS supplementary material at.

[48] B. Delley, B. J. Chem. Phys. 92, 508 (1990).

[49] B. Delley, J. Chem. Phys. 113, 7756 (2003).

[50] StoBe-deMon version 3.0 (2007), K. Hermann, L. G. M. Pettersson, M. E. Casida, C. Daul, A. Goursot, A. Koester, E. Proynov, A. St-Amant, D. R. Salahub. Contributing authors: V. Carravetta, H. Duarte, C. Friedrich, N. Godbout, J. Guan, C. Jamorski, M. Leboeuf, M. Leetmaa, M. Nyberg, S. Patchkovskii, L. Pedocchi, F. Sim, L. Triguero, A. Vela.

[51] A. D. Beche, Phys. Rev. A 38, 3098 (1988).

[52] J. P. Perdew, Phys. Rev. B 33, 8822 (1986).

[53] W. Kutzelnigg, U. Fleischer and M. Schindler, NMR-Basic Principles and Progess (SpringerVerlag, Heidelberg, 1990), Vol. 23, p165.

[54] B. Brena and Y. Luo, J. Chem. Phys. 119, 7139 (2003).

[55] J. Ahlund, K. Nilson, J. Schiessling, L. Kjeldgaard, S. Berner, N. Martensson, C. Puglia, B. Brena, M. Nyberg and Y. Luo, J. Chem. Phys. 125, 034709 (2006).

[56] W. Zhang, V. Carravetta, O. Plekan, V. Feyer, R. Richter, M. Coreno and K. C. Prince, J. Chem. Phys., 131, 035103(2009).

[57] Clar E. The Aromatic Sextet; Wiley-VCH: London, 1972. 